\title{
Poly(ADP-ribose) polymerase 1 in regulation of DNA repair and longevity
}

\author{
O.I. Lavrik \\ Institute of Chemical Biology and Fundamental Medicine SB RAS, Novosibirsk, Russia \\ Novosibirsk State University, Novosibirsk, Russia \\ e-mail:lavrik@niboch.nsc.ru
}

Key words: PARP1, PARP2, poly(ADP-ribose), BER, NER, role of PARP1 in longevity

Motivation and Aim: The phenomenon of nicotinamide adenine dinucleotide (NAD+)dependent poly(ADP-ribosyl)ation catalyzed with PARP1 was discovered long time ago, but it is still unclear how this post-translational modification governs a multitude of cellular processes including DNA repair. When interacting with the damaged DNA, PARP1 catalyzes the synthesis of a long branched poly(ADP-ribose) polymer (PAR) by using $\mathrm{NAD}^{+}$as a substrate. PAR can be attached to the acceptor amino acid residues of nuclear proteins or to PARP1 itself. This process leads to reorganization of the functional protein complexes involved in base excision repair (BER) and in nucleotide excision repair (NER). The aim of the present research was to investigate the role of poly(ADP-ribosyl)ation in regulation of BER and NER and to search new targets of PARylation catalyzed with PARP1 and PARP2. The activities of BER and NER systems were investigated in cells of long-living animal Heterocephalus glaber (H. glaber) in comparison with cells of Mus musculus (mouse).

Methods: Biochemical and immunochemical approaches, RT-PCR, fluorescence titration methods, atomic force microscopy (AFM), light-scattering technique.

Results: PARP1 interacts with BER and NER proteins as well as with DNA intermediates of BER containing breaks or apurinic/apyrimidinic (AP-sites). PARP1 and PARP2 activities regulate BER and NER processes. The new mechanism of stimulation of PARP1 activity by multifunctional protein YB-1 which involved in BER was found. The level of poly(ADP-ribose) synthesis was higher in the cells of H. glaber in comparison with mouse cells as well as $\mathrm{H}$. glaber cell extracts displayed higher level of PARP1 covalent binding to DNA probes containing chemically reactive groups.

Conclusion: The results obtained show the key role of PARP1 in regulation of BER and NER processes and the new mechanisms of stimulation of PARP1 activity by oncoprotein YB-1. The data show the elevated activity of PARP-1 in cells of long-living mammals that can speak in favor of the various roles of PARP1 in longevity and aging. Acknowledgements: This work was supported by grant from RSF (14-24-00038). 